\title{
¿Tienen los Hábitos de Vida y de Alimentación Impacto en la Calidad Seminal?
}

\section{Do Lifestyle and Eating Habits have an Impact on Seminal Quality?}

\author{
Alejandra María Gómez Gutiérrez ${ }^{1,2}$ Briana D. Gómez Ramírez ${ }^{3}$ Walter D. Cardona Maya10 \\ ${ }^{1}$ Grupo de Reproducción, Departamento de Microbiología y \\ Parasitología, Facultad de Medicina, Universidad de Antioquia, \\ Medellín, Colombia \\ 2 Departamento de Fisiología y Bioquímica, Facultad de Medicina, \\ Universidad de Antioquia, Medellín, Colombia \\ ${ }^{3}$ Escuela de Nutrición y Dietética, Universidad de Antioquia, Medellín, \\ Colombia \\ Dirección para correspondencia Walter D. Cardona Maya, Bact, MSc, \\ PhD, Grupo Reproducción, Departamento de Microbiología y \\ Parasitología, Facultad de Medicina, Universidad de Antioquia, \\ Carrera 53 \# 61-30. Sede de Investigación Universitaria, SIU; \\ Laboratorio 534, Medellín, Antioquia, Colombia \\ (e-mail: wdario.cardona@udea.edu.co).
}

Urol Colomb 2020;29:103-110.

\section{Resumen \\ Palabras claves \\ - vegetarianismo \\ - veganismo \\ - calidad seminal \\ - omnívoro \\ - fertilidad}

En los últimos años, se ha reportado en la literatura científica un declive en la calidad seminal. La significancia biológica de ese evento podría estar relacionada con la reducción en la fertilidad masculina o con el aumento del riesgo de enfermedades en la descendencia. La occidentalización en el estilo de vida, caracterizado por el aumento del peso corporal, el sedentarismo, el uso de cigarrillo, la ingesta de alimentos ricos en grasas y sodio y la reducción en la ingesta de alimentos fuente de fibras y antioxidantes, pudieran estar implicados en esos resultados. A su vez, cambios en el estilo de vida, que conduzcan a una reducción de peso corporal, de la ingesta de grasa y sodio, a un mejor estado antioxidante y a un aumento de la ingesta de fibra, se han asociado a mejores parámetros seminales. Por lo tanto, la alimentación basada en plantas o vegetariana, podría ser una estrategia válida para mejorar el estado nutricional y la salud masculina.

In recent years, a decline in seminal quality has been reported in the scientific literature. The biological significance of this event could be related to the reduction in male fertility or the increased risk of diseases in the offspring. Westernization in lifestyle, characterized by increased body weight, sedentary lifestyle, cigarette use, intake of foods high in fat and sodium and reduced intake of food sources of fiber and antioxidants, may be involved in these changes. Modifications in lifestyle, which lead to a reduction in body weight, fat and sodium intake, a better antioxidant status and an increase in fiber intake, have been associated with better seminal parameters. Therefore, plant-based or vegetarian food could be a valid strategy to improve nutritional status and male health. received

August 26, 2019

accepted

October 14, 2019
DOI https://doi.org/

$10.1055 / \mathrm{s}-0039-3402485$.

ISSN 0120-789X.

e ISSN 2027-0119.
Copyright $(2020$, Sociedad Colombiana License terms de Urología. Publicado por Thieme Revinter Publicações Ltda., Rio de Janeiro, Brazil. Todos los derechos reservados.

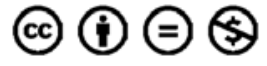




\section{Introducción}

Los estilos de vida inadecuados, caracterizados por el uso de licor, cigarrillo, inactividad física y malas prácticas alimentarias, se han propuesto como una de las principales causas de deterioro de la calidad seminal ${ }^{1,2}$ y se ha establecido que el entorno del padre proporciona gran parte de la herencia no genética, a través de la línea germinal, pues pudiera dar lugar a modificaciones epigenéticas en los espermatozoides, que pueden persistir en la descendencia. ${ }^{3}$

Los cambios ambientales a los que se expone el padre como los contaminantes, la dieta inadecuada, la obesidad, fumar, ingerir bebidas alcohólicas o exponerse a toxinas ocupacionales, tienen impacto en la metilación del DNA, en el incremento de las especies reactivas de oxígeno (ROS), en la modificación de histonas, y en la mayor cantidad de microRNA, ${ }^{4}$ lo cual puede conducir a una afectación negativa sobre la concentración, la movilidad y la morfología espermática, conduciendo inclusive a la imposibilidad para fecundar, alteración en el desarrollo embrionario y alteraciones en la descendencia que aumentan el riesgo de desarrollar enfermedades crónicas a futuro. 5,6

La evidencia anterior, hace pensar, que si los estilos de vida poco saludables, se asocian con el detrimento de la salud y de la calidad seminal, estilos de vida saludables en los que se evite el uso del cigarrillo y el licor, se incluya la práctica de actividad física, el descanso y la alimentación adecuadas, podrían tener un impacto positivo en la salud, en el peso y en la composición corporal y por ende, en la calidad seminal.

Las dietas vegetarianas han sido reconocidas desde hace mucho tiempo por conferir protección contra enfermedades como las cardiovasculares, ${ }^{7}$ inflamatorias, la diabetes mellitus tipo $2^{8,9}$ y el cáncer ${ }^{10}$ y contra factores de riesgo cardiometabólicos como la hipertensión $\operatorname{arterial}^{11}$ y la obesidad, ${ }^{12,13}$ pero el papel de la dieta vegetariana en la fertilidad masculina ha sido poco estudiado y la información aun es controversial.

Por lo tanto, con esta revisión de la literatura, se pretende recolectar y analizar la evidencia sobre la relación entre los hábitos y el estilo de vida y la calidad seminal, además, se revisa la evidencia con relación al impacto que tiene la práctica de un estilo de alimentación basado en plantas o vegetariano sobre los parámetros seminales.

\section{Situación de Salud y Estilo de Vida - Epidemiología Actual}

Las enfermedades crónicas no trasmisibles (ECNT), son enfermedades de larga duración y lenta evolución, que resultan de la combinación de factores genéticos, fisiológicos, ambientales y conductuales. ${ }^{14}$ Dentro de ellas, se encuentran las enfermedades cardiovasculares (ECV), como los infartos y los accidentes cerebrovasculares, el cáncer, las enfermedades respiratorias crónicas como la enfermedad pulmonar obstructiva crónica y la diabetes mellitus. La Organización Mundial de la Salud (OMS), reportó que aproximadamente 15 millones de todas las muertes atribuidas a las ECNT se producen prematuramente es decir, en personas entre los 30 y los 69 años de edad. ${ }^{15}$

El desarrollo de las ECNT se ve favorecido por factores tales como la urbanización rápida y no planificada, el envejecimiento de la población y la globalización de estilos de vida poco saludables caracterizados por dietas ricas en azúcares, sodio, grasa saturada, grasa trans y pobres en fibra y grasas insaturada, inactividad física, exposición al humo del tabaco o el uso nocivo del alcohol. ${ }^{15}$ En ese sentido, sabemos que casi 4,1 millones de muertes anuales se atribuyen a una ingesta excesiva de sodio, 3,3 millones de muertes anuales al consumo de alcohol, unas 1,6 millones de muertes anuales a una actividad física insuficiente y el tabaco es responsable de 7,2 millones de muertes al año. ${ }^{16}$

De acuerdo con el Estudio Nacional de Consumo de Sustancias Psicoactivas, para 2013 en Colombia, se ha reportado que la edad promedio de inicio de consumo de alcohol es a los 12 años de edad y la prevalencia en el consumo en los hombres adultos es de $56,7 \%$. De la misma manera el 42,1\% de los encuestados declaró haber consumido tabaco en algún momento de su vida, siendo un $71 \%$ mayor la prevalencia en hombres que en mujeres. Ese mismo estudio, exploró el uso de sustancias psicoactivas ilegales (marihuana, cocaína, bazuco, éxtasis, heroína, morfina -sin prescripción médica-, alucinógenos -LSD-, hongos) y encontró que el $13 \%$ de los encuestados han consumido alguna de ellas en determinado momento de su vida. La prevalencia año es 3,12 veces mayor en hombres que en mujeres. ${ }^{17}$ De otro lado, los datos de la Encuesta Nacional de la Situación Nutricional 2015 (ENSIN), reportan que en Colombia el $77 \%$ de los hombres adolescentes pasa un tiempo excesivo frente a las pantallas de aparatos electrónicos y en los adultos, solo el 61\% cumple las recomendaciones de actividad física. ${ }^{18}$

La práctica de éstos hábitos de estilo de vida inadecuados, ha llevado a que la obesidad en las personas adultas aumente; en Colombia se reporta un 18,7\% de adultos obesos y un $53 \%$ de hombres con sobrepeso y obesidad. ${ }^{18}$ Posiblemente asociado al aumento de la obesidad, la prevalencia de otras condiciones como la dislipidemia, la hipertensión arterial y la diabetes mellitus, también van en aumento. De acuerdo con la Encuesta Nacional de Salud, en Colombia la prevalencia de colesterol total mayor de $240 \mathrm{mg} /$ dL es de 7,8\%, de hipertensión arterial es de $22,8 \%$ y para el 2008 , en Colombia la prevalencia de hiperglucemia en ayunas ( $\geq 126 \mathrm{mg} / \mathrm{dL}$ ) en adultos de 25 o más años, fue de $6,7 \%$ en hombres. ${ }^{19}$

Preocupan esas cifras tan alarmantes con relación a la salud y el estilo de vida masculino, debido a que recientemente, como complemento al DOHad (Developmental Origins of Health and Disease) y dándole valor al factor masculino, se ha establecido el paradigma POHad (Paternal Origins of Health and Disease), en el cual se establece que los factores ambientales pre-concepcionales a los que se expone el padre, toman un papel muy importante en los cambios epigenéticos de los espermatozoides, cambios incluso asociados con la infertilidad o el desarrollo de enfermedades en el hijo. ${ }^{3}$ 


\section{Estilo de Vida Poco Saludable y Calidad Seminal}

En las últimas décadas, se ha presentado un aumento muy importante en la incidencia de algunas condiciones asociadas a la reproducción masculina, dentro de las que se encuentran la mala calidad del semen como lo demostró Carlsen y col., en 1990 que, mediante un meta análisis, reportaron una disminución en la concentración espermática, la movilidad y la morfología normal. ${ }^{20}$ Adicionalmente, un estudio longitudinal $^{21} \mathrm{y}$ otro transversal $^{22}$ realizados en Israel, demostraron una disminución significativa de la concentración y la movilidad, y un análisis retrospectivo realizado en Bélgica determino la disminución de la movilidad espermática. ${ }^{23}$ Por lo tanto, surge la preocupación de que sean ciertos productos químicos ambientales y algunos factores relacionados con el estilo de vida los responsables, en parte, de esos resultados. ${ }^{24}$

Borges y col., demostraron que fumar influye negativamente en el volumen del semen, en la concentración espermática tanto por $\mathrm{mL}$ como total y que el consumo de alcohol influye negativamente en la concentración de espermatozoides por $\mathrm{mL}$. Además, el éxito gestacional durante los procedimientos de reproducción asistida también fue negativamente influenciado por el consumo de alcohol y cigarrillo. Esas afectaciones pudieron haber estado relacionadas con el aumento de la injuria a los espermatozoides por el aumento en la cantidad de ROS y por el daño al DNA. ${ }^{2}$

Adicionalmente, es conocido que el índice de masa corporal (IMC) elevado en los hombres tiene efectos nocivos sobre los parámetros de fertilidad como lo demostraron Sermondade y col., en un meta análisis en el cual encontraron un mayor riesgo de alteraciones en la concentración espermática en hombres obesos o con sobrepeso. Como causa de esos cambios, se consideraron la alteración del eje hipotálamo-hipófisisgónadas o la aromatización de los esteroides masculinos a estrógenos en los tejidos periféricos que conduce a un hipogonadismo hiperestrogénico en esos pacientes. ${ }^{25}$ Recientemente, nuestro grupo determinó un mejoramiento en diferentes parámetros de la calidad seminal como volumen, concentración y movilidad en un hombre obeso con un IMC de $36,1 \mathrm{~kg} / \mathrm{mt}^{2}$ inicial, que se sometió a un plan para pérdida de peso y alcanzó un IMC de $29,9 \mathrm{~kg} / \mathrm{mt}^{2}{ }^{26}$ Incluso se ha demostrado una asociación entre la obesidad paterna y cambios en la metilación del DNA en los descendientes. Soubry y col., ${ }^{27}$ encontraron alteraciones en la metilación de múltiples regiones reguladoras en el DNA en niños nacidos de padres obesos, en comparación con los niños nacidos de padres no obesos. Esos datos sugieren una influencia preconcepcional del estilo de vida de los padres en la programación durante la gametogénesis y el desarrollo temprano de manera similar a lo que se ha demostrado sobre las alteraciones de la impronta materna, su transmisión a la próxima generación y el aumentó en el riesgo de enfermedades crónicas en la edad adulta. ${ }^{28}$

La dieta ha sido examinada con miras a determinar su impacto en la calidad seminal, pero los hallazgos no han sido concluyentes. Araba y col., en un meta análisis, determinaron una menor concentración de espermatozoides en voluntarios con una pobre adherencia a un patrón de dieta saludable, pero no encontraron otras asociaciones entre la alimentación y la calidad seminal. ${ }^{1}$ Salas-Huertas y col., ${ }^{29}$ por su parte, describieron que dietas ricas en nutrientes como ácidos grasos omega 3, antioxidantes, vitaminas y bajas en ácidos grasos saturados, ácidos grasos trans, son inversamente asociadas con bajos parámetros seminales y alimentos como pescados, comida de mar, cereales, vegetales y frutas son positivamente asociados con calidad espermática. Mientras que dietas ricas en alimentos procesados, carne roja, productos de soya y productos lácteos ricos en grasa son inversamente asociados con calidad seminal. ${ }^{29}$

Por su parte, Afeiche y col., ${ }^{30}$ investigaron la asociación de la ingesta de un grupo de alimentos, particularmente lácteos y la calidad seminal en un grupo de hombres que asistían a una clínica de fertilidad. Encontraron que los lácteos bajos en grasa se asociaron con una alta concentración de espermatozoides y mayor movilidad. Esa asociación fue independiente del tipo de dieta que seguían los hombres estudiados. ${ }^{30}$ Adicionalmente, la soya y alimentos derivados, se han asociado con una menor concentración espermática. Isoflavonas presentes en la soya, como la gliciteina pudieran estar asociadas a esos resultados. ${ }^{31}$

Estudios dirigidos a revisar el impacto de un nutriente en particular en la calidad seminal, tampoco han sido concluyentes. Dadkhah y col., mostraron que el aumento de $1 \mathrm{~g}$ en la ingesta de grasa total aumentaba el volumen del eyaculado hasta en un $27 \%$, mientras que el aumento en la ingesta de $1 \mathrm{~g}$ de grasa saturada lo reducía hasta en un $38 \%$, pero no se afectaban otras características de la calidad seminal como la movilidad, la concentración y la morfología. ${ }^{32}$ Algunas revisiones de la literatura han reportado como algunos nutrientes tienen un impacto positivo en la calidad seminal. La vitamina B12 parece incrementar el conteo de espermatozoides, la movilidad y reducir el daño al DNA, efectos atribuidos al papel que tiene el nutriente en la reducción de la homocisteína, a una reducción en la producción de óxido nítrico y de daños oxidativos y a una menor activación del NFkB. ${ }^{33}$

\section{Vegetarianismo y Efectos Sobre la Salud}

El Vegetarianismo es un término amplio que abarca una gama diversa y heterogénea de prácticas alimentarias e incluso tiene que ver con una identidad y estilo de vida. ${ }^{34}$ En general, una persona vegetariana es aquella que no consume alimentos de origen animal como carnes de res, aves, comida de mar o ingredientes que contengan esos alimentos de manera parcial o total, variando considerablemente los patrones de alimentación entre vegetarianos. Las dietas vegetarianas se pueden subcategorizar. Lacto-ovo-vegetariano que es aquella persona que come lácteos y huevos y sus derivados, como productos de origen animal, excluyendo únicamente la carne y sus derivados. Los lacto-vegetarianos suprimen adicionalmente los huevos y los veganos o vegetarianos totales, excluyen cualquier alimento, derivado e ingrediente de origen animal. ${ }^{35}$

Con el avance de los estudios y los cambios en los estilos de vida, aparecen clasificaciones cada vez más refinadas, como los pesco-vegetarianos, que incluyen pescados, pero no otras 
carnes y semi-vegetarianos que incluyen ocasionalmente (menos de 1 vez por semana), pescado o carnes blancas, entre otros productos de origen animal. ${ }^{36}$ También el flexitariano que es la persona que decide reducir el consumo de todo tipo de carnes, con fines casi siempre ambientales. ${ }^{37}$

Ese tipo de dietas presentan un perfil alimentario caracterizado por una mayor ingesta de granos enteros, frutas, soya y derivados, leguminosas y legumbres, vegetales, especialmente hojas verdes oscuras, nueces, semillas y en algunos casos mayor ingesta de leche y lácteos bajos en grasa, lo que la hace nutricionalmente adecuada. Puede aportar -bajo una correcta planeación- un alto contenido de fibra, magnesio, potasio, vitamina $\mathrm{A}$, vitamina $\mathrm{C}$, vitamina $\mathrm{E}$, tiamina, riboflavina, folatos, carotenoides, flavonoides y otros fitoquímicos de gran potencial antioxidante y dependiendo del tipo de vegetarianismo, aportará también suficiente cantidad de hierro y calcio, al tiempo que es baja en grasa saturada, colesterol, sodio, lo que puede explicar algunas de las ventajas que presentan para la salud. ${ }^{38,39}$ La ingesta de proteína, si bien pudiera ser menor que la de personas omnívoras, alcanza a cubrir los aportes recomendados para ese nutriente. ${ }^{39}$

Los diferentes tipos de dieta vegetariana y el tiempo de seguimiento de la misma, hacen difícil interpretar los resultados de los estudios y podrían explicar en algunos casos las discrepancias entre los mismos, pero a pesar de ello, con frecuencia en la literatura científica (meta-análisis y revisiones sistemáticas), las dietas vegetarianas se han asociado con ventajas para la salud, ${ }^{40-42}$ incluyendo la reducción en los valores de colesterol, menor estado inflamatorio, menor riesgo de enfermedades cardiovasculares y diabetes tipo 2, menor presión arterial, menor mortalidad por cardiopatía isquémica, menor riesgo de hipertensión y menor incidencia general de cáncer. $^{35,36,43}$ Inclusive, algunos ensayos han demostrado resultados satisfactorios del uso de dietas vegetarianas como parte del tratamiento de la artritis reumatoide. ${ }^{44}$ En contraste, los estudios que evalúan el efecto de la carne roja o procesada en los resultados de la salud, han demostrado un mayor riesgo de mortalidad total, mortalidad por enfermedad cardiovascular, mortalidad por cáncer, diabetes mellitus tipo 2 y cáncer de mama, esófago, estómago, colon, próstata y vejiga. ${ }^{45-50}$

Dentro de los mecanismos que podría explicar los efectos atribuidos a esas dietas están: $i$ ) mayor capacidad antioxidante y por lo tanto mejor estado antioxidante, ${ }^{51}$ eso se debe a que su dieta contiene más antioxidantes como vitamina $\mathrm{C}$, vitamina $\mathrm{E}$ y b-caroteno ${ }^{52}$; ii) menor ingesta de grasa, lo que se asocia con menor acumulación de lípidos intracelulares y menor resistencia a la insulina ${ }^{53}$; iii) ingesta de fibra que puede reducir la velocidad de absorción de la glucosa y de la grasa por el intestino, lo que reduce el índice glicémico de los carbohidratos, también algunos tipos de fibra se han asociado con mayor excreción de ácidos biliares. Ese fenómeno incrementa la secreción del péptido similar al glucagón 1 y mejora el control glicémico ${ }^{53}$; iv) menor ingesta de calorías, lo que contribuye a un menor peso corporal. Diferentes investigadores han encontrado que los vegetarianos son más delgados y tienen menor concentración de colesterol LDL y colesterol total ${ }^{43,54} ; v$ ) aunque aún no hay consenso acerca de si la microbiota intestinal es un ecosistema estable, resistente al cambio o si dietas nuevas pueden facilitar un cambio adaptativo rápido. Se han publicado trabajos presentando evidencia de una rápida adaptación cuando se asumen dietas basadas en plantas. La evidencia indica que cambios en la flora intestinal, modifican la cantidad y el tipo de sustancias bacterianas producidas y absorbidas por la mucosa intestinal. Esas sustancias, pueden influenciar procesos inflamatorios y la fermentación de la fibra por las bacterias intestinales, incrementa la producción de ácidos grasos de cadena corta, los cuales son una fuente energética para enterocito. ${ }^{55,56}$

\section{Dietas Basadas en Plantas y Calidad Seminal}

Los estudios que intentan relacionar el vegetarianismo y la calidad seminal son contradictorios y aun no permiten dilucidar si la dieta vegetariana tiene efectos positivos o negativos en la calidad seminal.

Estudios pioneros desarrollados en India, país con un alto porcentaje de población vegetariana y altas tasas de fertilidad, concluyeron que no había afectación de la calidad seminal. Arora y col., ${ }^{57}$ en 1961 encontraron que la concentración espermática y el porcentaje de espermatozoides móviles no era inferior en sujetos vegetarianos infértiles respecto a los hombres no vegetarianos sanos y concluyó que la espermatogénesis en ese grupo de hombres no era afectada por su tipo de alimentación. ${ }^{57}$ Por su parte Bhushan y col., en 1978, no reportaron diferencias significativas en parámetros como el volumen, la movilidad y la morfología entre individuos vegetarianos y no vegetarianos sanos. ${ }^{58}$

Más recientemente, un estudio examinó el efecto de la dieta vegetariana en la fertilidad masculina en vegetarianos que vivían en California, particularmente en la zona conocida como Loma Linda. El análisis de 474 hombres que incluían lacto-ovo vegetarianos, veganos y no vegetarianos permitió determinar que los lacto-ovo vegetarianos y veganos tenían una concentración espermática y una movilidad más baja. Los veganos además tenían un menor porcentaje de hiperactivación. Aspectos como la morfología, la integridad en la cromatina y la progresión, fue similar entre ambos grupos. ${ }^{59}$ En ese mismo sentido Liu y col., ${ }^{60}$ reportaron que no había relación entre una dieta más sana y la calidad del semen y que lacto-ovo vegetarianos tuvieron significativamente menores concentraciones de espermatozoides y movilidad total en comparación con hombres no vegetarianos. ${ }^{60}$ Se ha propuesto que la mayor ingesta de productos de soya, ricos en fitoestrógenos, podrían estar afectando la producción de hormonas masculinas $\mathrm{y}$ por lo tanto, reduciendo la concentración de testosterona, lo que pudiera explicar los resultados encontrados. ${ }^{59}$

Si bien la dieta mediterránea es un patrón alimentario que ocasionalmente incluye carne roja y más frecuentemente productos de mar, es un tipo de dieta rico en la ingesta de vegetales, granos, frutas, nueces, semillas, con beneficios bien establecidos $y$ asociados a fuertes efectos antiinflamatorios y antioxidantes. Karayiannis y col., ${ }^{61}$ en 2017, evaluaron la calidad seminal 225 hombres que iniciaron un patrón de dieta Mediterráneo y que asistían a 
una clínica de fertilidad. Encontraron que cuanto más adherencia había a la dieta mediterránea, había también una mejor calidad seminal: concentración por $\mathrm{mL}$, concentración total y movilidad progresiva. En ese tipo de dieta, el aporte de ácidos grasos omega 3 puede ser decisivo, dado que durante la maduración de los espermatozoides, los aportes de esos ácidos grasos a la membrana celular son muy importantes. $^{61}$ Dentro de los posibles mecanismos responsables de los mejores resultados, se considera la naturaleza anti-inflamatoria de esa dieta. La inflamación puede afectar las estructuras que forman parte del sistema reproductor y las glándulas accesorias. Por su parte Gaskins y col.; mostraron que la adhesión a un patrón de dieta con pescado, pollo, frutas, verduras y granos se asoció con un mayor porcentaje de esperma progresivamente móvil. ${ }^{62}$

Roos y colaboradores, ${ }^{63}$ en una revisión de ensayos clínicos, determinaron que 14 de 17 ensayos (82\%) analizados mostraron una mejoría en la calidad del esperma o tasa de embarazo después de una terapia con antioxidante, si bien en los artículos se habla más de suplementación, la dieta vegetariana es reconocida por su aporte considerable de sustancias protectoras contra daño oxidativo. ${ }^{63}$ Los antioxidantes presentes retardan o evitan el daño oxidativo causado por agentes como el anión superóxido, el peróxido de hidrógenos y otros radicales a los espermatozoides, previniendo así la aglutinación.

De otro lado, los marcadores inflamatorios como la interleuquina (IL) -6, IL-8, IL-18 y la proteína C reactiva, se han relacionado con la disfunción endotelial y futuros eventos cardiovasculares, ${ }^{64,65}$ e incluso han sido asociados positivamente con alteración del endotelio, del óxido nítrico y la disfunción eréctil, principalmente en hombres obesos, ${ }^{66}$ mientras que se ha observado la disminución significativa de la IL-6 y la proteína C reactiva en hombres sometidos a intervenciones dietéticas ${ }^{67-69}$ y mejor calidad de vida. Esos hallazgos, permiten pensar que las modificaciones e intervenciones en el estilo de vida ayudan a resolver los problemas de la disfunción eréctil probablemente a través de la reducción de los marcadores inflamatorios y la posterior mejora de la función endotelial, repercutiendo positivamente en una mejor calidad de vida. ${ }^{70}$

\section{Ácidos Grasos Poliinsaturados Omega 3 Como Determinantes de la Calidad Seminal}

Los testículos son un tejido con un importante metabolismo de ácidos grasos poliinsaturados, comparable con tejidos como el hígado o las neuronas; pero a diferencia de ellos, los testículos permanentemente agotan sus reservas de ácidos grasos poliinsaturados, debido a que son utilizados para ser incorporados en los espermatozoides, los cuales terminado su proceso de maduración, abandonan el testículo. ${ }^{71}$ Los espermatozoides humanos se caracterizan por presentar una alta proporción de ácidos grasos polinsaturados en las membranas celulares, especialmente ácidos grasos de 20 y 22 carbonos. La proporción de ácidos grasos omega 6 y 3 en las membranas celulares del espermatozoide, parece estar asociada a la calidad seminal, habiendo una mayor necesidad de ácidos grasos omega 3, pues se ha logrado determinar una incrementada actividad en la conversión hacia ácidos grasos de cadena más larga y desaturada, siendo el acido docosahexaenoico (DHA, C22:6 n-3) quien pudiera tener una implicación fundamental en la fertilidad. ${ }^{71}$ Altas cantidades de DHA son concentradas en la cabeza del espermatozoide humano y eso pudiera tener implicaciones en la fluidez y flexibilidad de la membrana y por lo tanto en la morfología de la cabeza del espermatozoide, en la reacción acrosomal y la fusión de membranas con el gameto femenino. ${ }^{71}$

Se ha documentado, como la dieta influencia el perfil de ácidos grasos que exhibe el espermatozoide. Estudios en jabalíes alimentados con dietas que tienen diferente relación de ácidos grasos omega 6 y omega 3, han permitido determinar que la mejor relación es 1:1, dado que jabalíes alimentados con esa proporción reportaron mayor desarrollo testicular, mayor número de espermatozoides, menores alteraciones morfológicas y en el plasma seminal tenían mayor concentración de fructosa y mayor actividad de enzimas como súper óxido dismutasa y glutatión peroxidasa. ${ }^{72}$ Chavarro y col., determinaron que la concentración de ácidos grasos trans presente en espermatozoides humanos de hombres infértiles, se asociaba inversamente con la concentración total de los mismos. A pesar de que en ese grupo no se evaluó la ingesta de alimentos, ese resultado se tomó como un biomarcador de ésta, puesto que el metabolismo humano de las grasas no introduce enlaces en forma trans a los ácidos grasos y por lo tanto, su presencia en cualquier tejido o tipo de célula, en este caso en los espermatozoides, implica que fueron aportados por la dieta. ${ }^{73}$ Attaman y col., por su parte, relacionaron negativamente el alto consumo de grasas saturadas con la concentración de esperma, mientras que una mayor ingesta de grasas omega-3 lo relacionó positivamente con la morfología de los espermatozoides. ${ }^{74}$

Se ha sugerido que la síntesis de DHA a partir del alfa linolenico es ineficiente y eso es de particular relevancia en sujetos vegetarianos y veganos, porque ese ácido graso parece ser escaso o ausente en ese tipo de dietas. ${ }^{39}$ Revisiones sistemáticas de la literatura han determinado que a pesar de la presencia de alfa linolenico; ni DHA ni eicosapentaenoico (EPA) están presentes en dietas vegetarianas y que además la cantidad de ácido graso linoleico es considerablemente mayor que en los omnívoros. En ese escenario, la competencia existente entre esos dos tipos de ácidos grasos por las enzimas encargadas de la desaturación y elongación de los mismos, suprimirá la síntesis de DHA y favorecerá la producción de ácidos grasos de la familia omega 6. La transformación de alfa linolenico en DHA se produce a través de múltiples reacciones de desaturación y elongación, la cual no es eficiente en el cuerpo humano y varía según el sexo. ${ }^{75}$ En los hombres, la tasa de conversión de alfa linolenico a EPA es aproximadamente del $8 \%$, mientras que alfa linolenico a DHA está entre el $0 \%$ y el $4 \%$, valores que también dependen de la condición fisiológica. Esos eventos hacen pensar que la posible inadecuación en la relación de ingesta de ácidos grasos omega 6 y omega 3 que puede presentarse en vegetarianos, podría ser una desventaja para ellos. ${ }^{76}$ 


\section{Conclusión}

No queda claro si la dieta vegetariana tiene efectos en la calidad seminal debido a que los resultados son contradictorios. El impacto de la dieta vegetariana en la calidad seminal es complejo de analizar porque el vegetarianismo es un término diverso que puede implicar solo un cambio alimentario, permaneciendo con otro tipo de prácticas inadecuadas para la calidad seminal o transformado el estilo de vida adicional al cambio del consumo de alimentos, que incluso podría conducir a beneficios en la salud y a mucho más en el estado de salud de la descendencia.

\section{Conflicto de Intereses}

Los autores declaran no tener ningún conflicto de intereses.

\section{Referencias}

1 Arab A, Rafie N, Mansourian M, Miraghajani M, Hajianfar $\mathrm{H}$. Dietary patterns and semen quality: a systematic review and meta-analysis of observational studies. Andrology 2018;6(01): 20-28

2 Borges E Jr, Braga DPAF, Provenza RR, Figueira RCS, Iaconelli A Jr, Setti AS. Paternal lifestyle factors in relation to semen quality and in vitro reproductive outcomes. Andrologia 2018;50(09): e13090

3 Soubry A. POHaD: why we should study future fathers. Environ Epigenet 2018;4(02):dvy007

4 Macartney EL, Crean AJ, Bonduriansky R. Epigenetic paternal effects as costly, condition-dependent traits. Heredity 2018;121 (03):248-256

5 Navarro E, Funtikova AN, Fíto M, Schröder H. Prenatal nutrition and the risk of adult obesity: Long-term effects of nutrition on epigenetic mechanisms regulating gene expression. J Nutr Biochem 2017;39:1-14

6 Sanchez-Garrido MA, Ruiz-Pino F, Velasco I, et al. Intergenerational Influence of Paternal Obesity on Metabolic and Reproductive Health Parameters of the Offspring: MalePreferential Impact and Involvement of Kiss1-Mediated Pathways. Endocrinology 2018;159(02):1005-1018

7 Lin TJ, Tang SC, Liao PY, Dongoran RA, Yang JH, Liu CH. A comparison of L-carnitine and several cardiovascular-related biomarkers between healthy vegetarians and omnivores. Nutrition 2019;66:29-37

8 Tonstad S, Stewart K, Oda K, Batech M, Herring RP, Fraser GE. Vegetarian diets and incidence of diabetes in the Adventist Health Study-2. Nutr Metab Cardiovasc Dis 2013;23(04):292-299

9 Cui X, Wang B, Wu Y, et al. Vegetarians have a lower fasting insulin level and higher insulin sensitivity than matched omnivores: A cross-sectional study. Nutr Metab Cardiovasc Dis 2019;29(05): 467-473

10 Gajski G, Gerić M, Vučić Lovrenčić M, et al. Analysis of healthrelated biomarkers between vegetarians and non-vegetarians: A multi-biomarker approach. J Funct Foods 2018;48:643-653

11 Garbett TM, Garbett DL, Wendorf A. Vegetarian Diet: A Prescription for High Blood Pressure? A Systematic Review of the Literature. J Nurse Pract 2016;12(07):452-458

12 Mihrshahi S, Ding D, Gale J, Allman-Farinelli M, Banks E, Bauman AE. Vegetarian diet and all-cause mortality: Evidence from a large population-based Australian cohort - the 45 and Up Study. Prev Med 2017;97:1-7

13 Jaceldo-Siegl K, Haddad E, Knutsen S, et al. Lower C-reactive protein and IL-6 associated with vegetarian diets are mediated by BMI. Nutr Metab Cardiovasc Dis 2018;28(08):787-794
14 Diet W. nutrition and the prevention of chronic diseases. Report of a joint WHO/FAO expert consultation. WHO. Tech Rep Ser (World Health Organ) 2003;916:34-38

15 World Health Organization. Global status report on noncommunicable diseases 2014. 2014https://www.who.int/ $\mathrm{nmh} /$ publications/ncd-status-report-2014/en/

16 World Health Organization. Noncommunicable diseases https:// www.who.int/news-room/fact-sheets/detail/noncommunicablediseases [Noncommunicable diseases].

17 Ods M, Minsalud CO. Estudio Nacional de consumo de sustancias psicoactivas en Colombia. 2013

18 Encuesta nacional de la Situación Nutricional 2015 http://www. prosperidadsocial.gov.co/temporales/

EncuestaNacionaldelaSituación\%20Nutricional\%20-\%20ENSIN\% 202015.pdf2015

19 Gobierno de Colombia. Ministerio de Salud y protección social Dirección de Epidemiología y Demografía. Analisis De Situación De Salud (ASIS) Colombia, 2017: https://www.minsalud.gov.co/ sites/rid/Lists/BibliotecaDigital/RIDE/VS/ED/PSP/asis-nacional2017.pdf; 2017

20 Carlsen E, Giwercman A, Keiding N, Skakkebaek NE. Evidence for decreasing quality of semen during past 50 years. BMJ 1992;305 (6854):609-613

21 Almagor M, Ivnitzki I, Yaffe H, Baras M. Changes in semen quality in Jerusalem between 1990 and 2000: a cross-sectional and longitudinal study. Arch Androl 2003;49(02):139-144

22 Haimov-Kochman R, Har-Nir R, Ein-Mor E, et al. Is the quality of donated semen deteriorating? Findings from a 15 year longitudinal analysis of weekly sperm samples. Isr Med Assoc J 2012;14(06):372-377

23 Van Waeleghem K, De Clercq N, Vermeulen L, Schoonjans F, Comhaire F. Deterioration of sperm quality in young healthy Belgian men. Hum Reprod 1996;11(02):325-329

24 Giwercman A, Giwercman YL. Environmental factors and testicular function. Best Pract Res Clin Endocrinol Metab 2011; 25(02):391-402

25 Sermondade N, Faure C, Fezeu L, et al. BMI in relation to sperm count: an updated systematic review and collaborative metaanalysis. Hum Reprod Update 2013;19(03):221-231

26 Puerta-Suárez J, Gómez-Gutiérrez A, Cardona-Maya W. Efecto de la disminución del índice de masa corporal sobre la calidad seminal. Rev Cuba Obstet Ginecol 2019;45(02):

27 Soubry A, Murphy SK, Wang F, et al. Newborns of obese parents have altered DNA methylation patterns at imprinted genes. Int J Obes 2015;39(04):650-657

28 Agarwal P, Morriseau TS, Kereliuk SM, Doucette CA, Wicklow BA, Dolinsky VW. Maternal obesity, diabetes during pregnancy and epigenetic mechanisms that influence the developmental origins of cardiometabolic disease in the offspring. Crit Rev Clin Lab Sci 2018;55(02):71-101

29 Salas-Huetos A, Bulló M, Salas-Salvadó J. Dietary patterns, foods and nutrients in male fertility parameters and fecundability: a systematic review of observational studies. Hum Reprod Update 2017;23(04):371-389

30 Afeiche MC, Bridges ND, Williams PL, et al. Dairy intake and semen quality among men attending a fertility clinic. Fertil Steril 2014; 101(05):1280-1287

31 Chavarro JE, Toth TL, Sadio SM, Hauser R. Soy food and isoflavone intake in relation to semen quality parameters among men from an infertility clinic. Hum Reprod 2008;23(11):2584-2590

32 Dadkhah H, Kazemi A, Nasr-Isfahani M-H, Ehsanpour S. The relationship between the amount of saturated fat intake and semen quality in men. Iran J Nurs Midwifery Res 2017;22(01):46-50

33 Banihani SA. Vitamin $B_{12}$ and Semen Quality. Biomolecules 2017; 7(02):E42

34 Cramer H, Kessler CS, Sundberg T, et al. Characteristics of Americans Choosing Vegetarian and Vegan Diets for Health Reasons. J Nutr Educ Behav 2017;49(07):561-567.e1 
35 Craig WJ, Mangels AR; American Dietetic Association. Position of the American Dietetic Association: vegetarian diets. J Am Diet Assoc 2009;109(07):1266-1282

36 Fraser GE. Vegetarian diets: what do we know of their effects on common chronic diseases? Am J Clin Nutr 2009;89(05): 1607S-1612S

37 Raphaely T, Marinova D. Flexitarianism: Decarbonising through flexible vegetarianism. Renew Energy 2014;67:90-96

38 Farmer B, Larson BT, Fulgoni VL III, Rainville AJ, Liepa GU. A vegetarian dietary pattern as a nutrient-dense approach to weight management: an analysis of the national health and nutrition examination survey 1999-2004. J Am Diet Assoc 2011;111(06): 819-827

39 Rizzo NS, Jaceldo-Siegl K, Sabate J, Fraser GE. Nutrient profiles of vegetarian and nonvegetarian dietary patterns. J Acad Nutr Diet 2013;113(12):1610-1619

40 Huang T, Yang B, Zheng J, Li G, Wahlqvist ML, Li D. Cardiovascular disease mortality and cancer incidence in vegetarians: a metaanalysis and systematic review. Ann Nutr Metab 2012;60(04): 233-240

41 Key TJ, Fraser GE, Thorogood M, et al. Mortality in vegetarians and nonvegetarians: detailed findings from a collaborative analysis of 5 prospective studies. Am J Clin Nutr 1999;70(3, Suppl) 516S-524S

42 Key TJ, Appleby PN, Spencer EA, Travis RC, Roddam AW, Allen NE. Cancer incidence in vegetarians: results from the European Prospective Investigation into Cancer and Nutrition (EPICOxford). Am J Clin Nutr 2009;89(05):1620S-1626S

43 Le LT, Sabaté J. Beyond meatless, the health effects of vegan diets: findings from the Adventist cohorts. Nutrients 2014;6(06): 2131-2147

44 Kjeldsen-Kragh J. Rheumatoid arthritis treated with vegetarian diets. Am J Clin Nutr 1999;70(3, Suppl)594S-600S

45 Lippi G, Mattiuzzi C, Cervellin G. Meat consumption and cancer risk: a critical review of published meta-analyses. Crit Rev Oncol Hematol 2016;97:1-14

46 Micha R, Wallace SK, Mozaffarian D. Red and processed meat consumption and risk of incident coronary heart disease, stroke, and diabetes mellitus: a systematic review and meta-analysis. Circulation 2010;121(21):2271-2283

47 Pan A, Sun Q Bernstein AM, et al. Red meat consumption and mortality: results from 2 prospective cohort studies. Arch Intern Med 2012;172(07):555-563

48 Pan A, Sun Q, Bernstein AM, et al. Red meat consumption and risk of type 2 diabetes: 3 cohorts of US adults and an updated metaanalysis. Am J Clin Nutr 2011;94(04):1088-1096

49 Sinha R, Cross AJ, Graubard BI, Leitzmann MF, Schatzkin A. Meat intake and mortality: a prospective study of over half a million people. Arch Intern Med 2009;169(06):562-571

50 Wolk A. Potential health hazards of eating red meat. J Intern Med 2017;281(02):106-122

51 Szeto YT, Kwok TC, Benzie IF. Effects of a long-term vegetarian diet on biomarkers of antioxidant status and cardiovascular disease risk. Nutrition 2004;20(10):863-866

52 Rauma AL, Mykkänen H. Antioxidant status in vegetarians versus omnivores. Nutrition 2000;16(02):111-119

53 Yokoyama Y, Barnard ND, Levin SM, Watanabe M. Vegetarian diets and glycemic control in diabetes: a systematic review and metaanalysis. Cardiovasc Diagn Ther 2014;4(05):373-382

54 Yokoyama Y, Levin SM, Barnard ND. Association between plantbased diets and plasma lipids: a systematic review and metaanalysis. Nutr Rev 2017;75(09):683-698

55 Glick-Bauer M, Yeh M-C. The health advantage of a vegan diet: exploring the gut microbiota connection. Nutrients 2014;6(11): 4822-4838
56 Moraes AC, de Almeida-Pittito B, Ferreira SRG. The Gut Microbiome in Vegetarians. In: Faintuch J, Faintuch S, editors. Microbiome and Metabolome in Diagnosis, Therapy, and other Strategic Applications: Academic Press; 2019:393-400

57 Arora RB, Saxena KN, Choudhury MR, Choudhury RR. Sperm studies on Indian men. Fertil Steril 1961;12:365-367

58 Bhushan S, Pandey RC, Singh SP, Pandey DN, Seth P. Some observations on human semen analysis. Indian $\mathrm{J}$ Physiol Pharmacol 1978;22(04):393-396

59 Orzylowska EM, Jacobson JD, Bareh GM, Ko EY, Corselli JU, Chan PJ. Food intake diet and sperm characteristics in a blue zone: a Loma Linda Study. Eur J Obstet Gynecol Reprod Biol 2016;203:112-115

60 Liu CY, Chou YC, Chao JC, Hsu CY, Cha TL, Tsao CW. The Association between Dietary Patterns and Semen Quality in a General Asian Population of 7282 Males. PLoS One 2015;10(07):e0134224

61 Karayiannis D, Kontogianni MD, Mendorou C, Douka L, Mastrominas M, Yiannakouris $N$. Association between adherence to the Mediterranean diet and semen quality parameters in male partners of couples attempting fertility. Hum Reprod 2017;32(01):215-222

62 Gaskins AJ, Colaci DS, Mendiola J, Swan SH, Chavarro JE. Dietary patterns and semen quality in young men. Hum Reprod 2012;27 (10):2899-2907

63 Ross C, Morriss A, Khairy M, et al. A systematic review of the effect of oral antioxidants on male infertility. Reprod Biomed Online 2010;20(06):711-723

64 Blake GJ, Ridker PM. Novel clinical markers of vascular wall inflammation. Circ Res 2001;89(09):763-771

65 Blankenberg S, Tiret L, Bickel C, et al; AtheroGene Investigators. Interleukin-18 is a strong predictor of cardiovascular death in stable and unstable angina. Circulation 2002;106(01):24-30

66 Giugliano F, Esposito K, Di Palo C, et al. Erectile dysfunction associates with endothelial dysfunction and raised proinflammatory cytokine levels in obese men. J Endocrinol Invest 2004;27(07):665-669

67 Esposito K, Ciotola M, Giugliano F, et al. Mediterranean diet improves erectile function in subjects with the metabolic syndrome. Int J Impot Res 2006;18(04):405-410

68 Esposito K, Giugliano F, Di Palo C, et al. Effect of lifestyle changes on erectile dysfunction in obese men: a randomized controlled trial. JAMA 2004;291(24):2978-2984

69 Khoo J, Piantadosi C, Duncan R, et al. Comparing effects of a lowenergy diet and a high-protein low-fat diet on sexual and endothelial function, urinary tract symptoms, and inflammation in obese diabetic men. J Sex Med 2011;8(10):2868-2875

70 Hannan JL, Maio MT, Komolova M, Adams MA. Beneficial impact of exercise and obesity interventions on erectile function and its risk factors. J Sex Med 2009;6(Suppl 3):254-261

71 Esmaeili V, Shahverdi AH, Moghadasian MH, Alizadeh AR. Dietary fatty acids affect semen quality: a review. Andrology 2015;3(03): 450-461

72 Lin Y, Cheng X, Mao J, et al. Effects of different dietary n-6/n-3 polyunsaturated fatty acid ratios on boar reproduction. Lipids Health Dis 2016;15:31

73 Chavarro JE, Furtado J, Toth TL, et al. Trans-fatty acid levels in sperm are associated with sperm concentration among men from an infertility clinic. Fertil Steril 2011;95(05):1794-1797

74 Attaman JA, Toth TL, Furtado J, Campos H, Hauser R, Chavarro JE. Dietary fat and semen quality among men attending a fertility clinic. Hum Reprod 2012;27(05):1466-1474

75 Burdge GC, Calder PC. Conversion of alpha-linolenic acid to longer-chain polyunsaturated fatty acids in human adults. Reprod Nutr Dev 2005;45(05):581-597

76 Sanders TA. DHA status of vegetarians. Prostaglandins Leukot Essent Fatty Acids 2009;81(2-3):137-141 
